\title{
Rassegna
}

\section{La prevenzione primaria e secondaria dell'ipertensione arteriosa: il punto di vista del nefrologo}

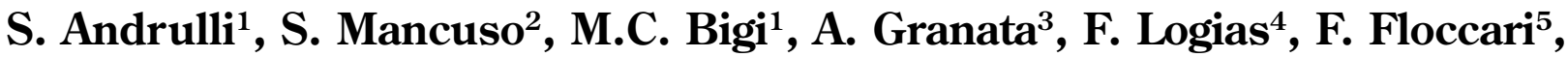 \\ L. Di Lullo ${ }^{6}$
}

${ }^{1}$ Divisione di Nefrologia, Dialisi, Ospedale 'Alessandro Manzoni', Lecco

${ }^{2}$ Centro Emodialisi Mazarese, Mazara del Vallo (Trapani)

${ }^{3}$ Divisione di Nefrologia e Dialisi, Ospedale 'San Giovanni di Dio', Agrigento

${ }^{4}$ Servizio di Dialisi, Ospedale 'San Camillo', Sorgono (Nuoro)

${ }^{5}$ Divisione di Nefrologia e Dialisi, Ospedale 'San Paolo', Civitavecchia

${ }^{6}$ Divisione di Nefrologia e Dialisi, Ospedale 'L. Parodi-Delfino', Colleferro (Roma)

PRIMARY AND SECONDARY PREVENTION OF ARTERIAL HYPERTENSION: NEPHROLOGIST'S POINT OF VIEW

AвSTRACt. Nephroangiosclerotic chronic kidney disease's primary and secondary prevention is actually based on blood pressure control and reduction of proteinuria. In proteinuric patients, blood pressure control has to be obtained by blocking renin-angiotensin axis, even in patients with moderate to severe chronic kidney disease. Combination therapy with angiotensin converting enzyme inhibitor (ACEi) drugs and angiotensin II receptor blockers (ARB) doesn't imply addictive protective effects. It could be tested in selected patients with proteinuria levels above $1 \mathrm{~g} /$ day. Combination therapy with ACEi and anti-aldosteronic agents seems to be promising but we need more multicentric trials.

Key Words. Primary and secondary prevention, Arterial hypertension, Global cardio-vascular risk, Drug compliance, Personalised therapy, Proteinuria

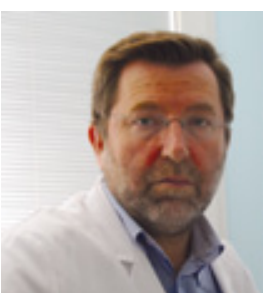

Simeone Andrulli

\section{Epidemiologia}

Prevenire è sicuramente meglio che curare. Questo è ancora più vero quando si è di fronte a un paziente con ipertensione arteriosa, visto che spesso l'ipertensione precede anche di molti anni la comparsa di un evento cardiovascolare rilevante, come un infarto del miocardio, un ictus o un ricovero per scompenso cardiaco o un evento renale su base vascolare. Come è noto, l'ipertensione arteriosa è un fenomeno multifattoriale in cui diversi fattori ambientali, come la dieta ipercalorica, l'introito di cibi salati, lo stress e la sedentarietà (tutti fattori connessi a uno stile di vita di tipo occidentale oppure occidentalizzato) interagiscono con fattori genetici, importanti nella sua forma più comune, ossia l'ipertensione arteriosa essenziale, idiopatica o primaria.

Malgrado la terapia antipertensiva, l'ipertensione arteriosa rappresenta ancora oggi il principale fattore di rischio per mortalità cardiovascolare nella popolazione ed è uno dei principali responsabili di morbilità e inabilità permanente. Questo spiega il motivo per cui l'identificazione delle persone a rischio cardiovascolare elevato sia uno degli obiettivi principali della prevenzione primaria, ossia prima che l'evento cardiovascolare si verifichi. L'ipertensione arteriosa interessa il 37\% degli italiani adulti e la sua prevalenza aumenta notevolmente con l'età soprattutto se ci riferiamo alla pressione arteriosa sistolica. Si stima che ogni anno in Italia circa 220000 ictus, 90000 infarti del miocardio e 
180000 casi di scompenso cardiaco siano il risultato di una ipertensione arteriosa non diagnosticata o scarsamente controllata.

\section{Diagnosi}

Lidentificazione precoce delle persone affette da ipertensione arteriosa costituisce un intervento efficace di prevenzione individuale e di comunità. Uno dei punti cardine delle linee guida europee dell'ipertensione arteriosa ESH/ESC pubblicate nel 2007 (1) è costituito dalla corretta identificazione del rischio cardiovascolare globale e dall'individuazione precoce di quei pazienti che necessitino di un approccio terapeutico immediato e/o più aggressivo.

L'elemento base per poter fare una diagnosi di ipertensione arteriosa è la precisa misurazione della pressione arteriosa effettuata sia con i sistemi di misurazione manuale, che richiedono una certa esperienza, sia con quelli elettronici a lettura automatica, di uso più semplice e praticabile comodamente al proprio domicilio. Utile osservare alcuni accorgimenti: si deve essere tranquilli e rilassati; la prima volta è opportuno fare una misurazione in clino ed ortostatismo e agli arti superiori e inferiori; in caso di riscontro di valori elevati, effettuare almeno 2 misurazioni a distanza di 2 minuti l'una dall'altra. Una volta accertata la presenza di ipertensione arteriosa è opportuno valutare sempre la presenza di possibili cause di ipertensione secondaria.

\section{Terapia}

L'obiettivo primario del trattamento del paziente iperteso è quello di ottenere la massima riduzione del rischio a lungo termine di mortalità e morbilità cardiovascolare. Tutto ciò richiede il trattamento di tutti i fattori di rischio identificabili e reversibili, che comprendono il sovrappeso, il fumo, la dislipidemia, il diabete nonché il trattamento degli elevati regimi pressori. Le modificazioni dello stile di vita sono imprescindibili in tutti i pazienti. Tuttavia, esse non sono sempre sufficienti e, pertanto, devono essere combinate con la terapia farmacologica, che va intesa quindi in senso additivo. Le classi farmacologiche principali sono i diuretici, gli ACE-inibitori, i sartani, i calcioantagonisti, i betabloccanti, gli alfalitici e i vasodilatatori. Tutte queste classi sono di prima scelta, ma alcune sono da preferirsi ad altre in particolari condizioni cliniche e/o in presenza di fattori di rischio cardiovascolare aggiuntivi. I farmaci antipertensivi oggi in commercio sono di solito sia efficaci sia ben tollerati.
Purtroppo non sempre è sufficiente un solo farmaco per ottenere un buon controllo pressorio. Spesso si deve ricorrere a una terapia di associazione fondata sull'assunzione di due o più farmaci. Alcune associazioni di farmaci antipertensivi sono già presenti in commercio come preparazioni precostituite, altre devono essere realizzate ricorrendo alla combinazione di più compresse. Esistono molte possibilità di combinazioni farmacologiche antipertensive, quali ad esempio quelle tra ace-inibitori o sartani con tiazidico.

\section{Compliance alla terapia}

L'ipertensione arteriosa continua a mietere vittime nonostante sia di facile diagnosi e nonostante la disponibilità di un numeroso e consistente armamentario terapeutico. I motivi di questo insuccesso terapeutico sono riassunti nella Tabella I. L'attenzione al consumo di sale, la perdita di peso e l'attività fisica costituiscono misure efficaci per ridurre i valori pressori, ma molte persone con ipertensione arteriosa richiedono anche un trattamento farmacologico. La scarsa compliance o adesione del paziente ai suggerimenti dietetici e di stile di vita come alla terapia prescritta, nonché il suo grado di cooperazione con il clinico nell'ambito di un programma terapeutico condiviso, rappresentano la causa principale di insuccesso terapeutico. Nella Tabella II sono indicati gli accorgimenti utili per migliorare la compliance alla

\section{TABELLA I - MOTIVI PER CUI L'IPERTENSIONE ARTERIO- SA CONTINUA A PRODURRE EVENTI CARDIO- VASCOLARI}

- $\quad$ Elevata prevalenza di ipertensione e sua lenta crescita

- Insufficiente controllo pressorio

- Scarsa attenzione a modificare lo stile di vita

- Coesistenza di fattori di rischio multipli

- La terapia farmacologica deve essere additiva alle modifiche dello stile di vita

- Scarsa compliance alla terapia

TABELLA II - COME MIGLIORARE LA COMPLIANCE ALLA TERAPIA

- L'informazione del paziente

- Ridurre lentamente la pressione arteriosa

- Buon rapporto tra medico e paziente

- Avere bene in mente il concetto del rischio cardiovascolare globale

- Personalizzare la terapia in base alla storia clinica del paziente

- Considerare gli effetti collaterali dei farmaci 
terapia. Linformazione al paziente va data in modo semplice ed efficace, nella forma più idonea considerando le sue caratteristiche anagrafiche, psicologiche e socio-sanitarie, per esempio ricorrendo alla sua storia familiare se positiva dal punto di vista cardiovascolare: per esempio. una madre che ha subito un ictus, un padre con storia di infarto, scompenso cardiaco o con insufficienza renale cronica hanno per il paziente un ruolo persuasivo maggiore di un'informazione vera ma astratta di un " $r i$ schio cardiovascolare globale". Dato che l'ipertensione arteriosa fa danni usualmente solo dopo anni, è sempre meglio ridurla a valori normali molto lentamente anche nel giro di alcune settimane. Conquistarsi la fiducia del paziente è fondamentale per assicurarlo sul risultato di un buon controllo pressorio che sarà raggiunto gradualmente, con calma (quindi eventualmente anche dopo qualche settimana) e soprattutto senza accusare effetti collaterali e/o indesiderati. Una normalizzazione rapida della pressione arteriosa farà sentire il paziente fiacco 'ipoteso' tanto da suggerirgli che la sua pressione arteriosa normale sia quella alta rilevata precedentemente! Il paziente deve sapere dal suo medico quanto sia importante raggiungere e mantenere un buon controllo pressorio anche mediante l'automisurazione a domicilio, quanto sia importante continuare la terapia antipertensiva per mantenere i livelli normali raggiunti e quali possono essere gli effetti collaterali dei farmaci. L'uso di un diario della pressione arteriosa e della frequenza cardiaca sarà di aiuto al paziente e al medico per variare e modulare la terapia nel modo più efficace. Se il paziente ha una certa età e concomita un certo sovrappeso, uno scompenso cardiaco o una riduzione della funzione renale, sarà molto utile associare un diario del peso, prima settimanale e poi mensile, e a quadro clinico stabilizzato.

\section{Terapia personalizzata}

La scelta dei farmaci sarà personalizzata sul paziente, in base alla sua storia clinica e alla conoscenza degli effetti collaterali dei farmaci. Quindi se il paziente è anziano e presenta qualche nota di scompenso cardiaco subclinico pensare all'utilità di un ace-inibitore o di un diuretico, magari in associazione soprattutto se il paziente non ama prendere troppe compresse. Se il paziente è giovane, sportivo e ha una certa vita di relazione cercare di escludere in prima battuta il betabloccante che sarà invece consigliato di fronte a un paziente con cardiopatia ischemica o pregresso infarto. Se un iperteso ha una proteinuria sopra al grammo/die e/o se è diabetico, sarà meglio dare la precedenza ad un ace-inibitore e/o sartano allo scopo di ridurre la progressione del danno

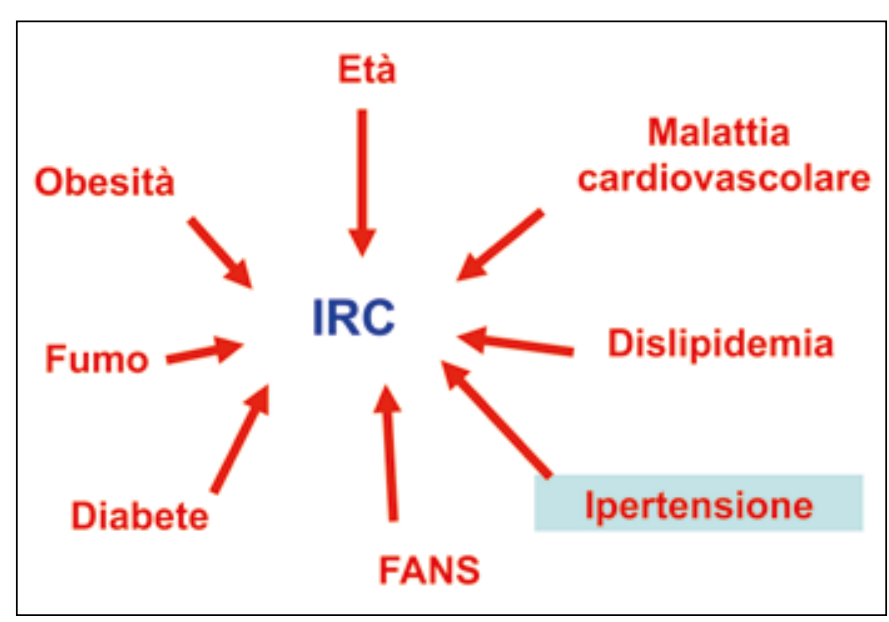

Fig. 1 - Fattori che influenzano la progressione dell'IRC.

renale. Se il paziente presenta una ipertrofia prostatica benigna con un certo residuo vescicale post-minzionale sarà meglio partire con un alfalitico. La terapia antipertensiva andrà comunque sempre personalizzata anche sulla base delle caratteristiche cliniche e psicologiche del paziente e modulata nello stesso paziente anche in base al susseguirsi delle stagioni, per i riflessi sulla pressione arteriosa della temperatura ambientale (spesso ma non sempre si abbassa d'estate) e delle variazioni stagionali della dieta.

\section{Terapia nel paziente con insufficienza renale cronica e/o proteinuria $>1 \mathrm{~g} / \mathrm{die}$}

Di fronte a un paziente con insufficienza renale cronica e/o proteinuria superiore al grammo/die occorre considerare da una parte il contesto multifattoriale alla base della progressione dell'insufficienza renale cronica (Fig. 1) e dall'altra il concetto di sinergia di effetti negativi sull'incidenza di eventi cardiovascolari quando l'ipertensione arteriosa sistolica e diastolica si associa a una riduzione della funzione renale: il rischio di eventi cardiovascolari aumenta di 3 volte se la funzione renale è ridotta sotto il $30 \%$ (filtrato stimato di $15-29 \mathrm{ml} / \mathrm{min}$ ) (2). Il 'goal 'sarà quindi da una parte mirare a un controllo pressorio più stretto (il valore desiderato diventa $120 / 80 \mathrm{mmHg}$ ) (3) e dall'altra considerare il ruolo preminente della proteinuria che aumenta di molto il rischio di progressione della malattia cronica renale (4) e ancora il maggior beneficio atteso nel paziente più proteinurico dallo stretto controllo pressorio (5) e dalla terapia con ace-inibitore (6). Se il paziente è anche diabetico, il farmaco di prima scelta diventa un sartano (7). Per massimizzare l'effetto 
anti-proteinurico, si può ricorrere alla terapia di associazione ace-inibitore/sartano (8) o ace-inibitore/antialdosteronico (9), ma il rischio di maggiori effetti collaterali negativi imporrà un monitoraggio più stretto del paziente dal punto di vista clinico e laboratoristico, per un maggior rischio di peggioramento funzionale renale, iperkaliemia o ginecomastia (quest'ultimo in caso di terapia con antialdosteronico). Il beneficio della terapia con ace-inibitore in termini di ridotta progressione del danno renale è mantenuto anche nei pazienti con importante insufficienza renale cronica (creatininemia compresa tra 3.1 e $5 \mathrm{mg} / \mathrm{dL}$ ) con il consiglio di uno stretto monitoraggio clinico e laboratoristico del paziente (10). Va sottolineato come anche l'utilizzo del tiazidico in associazione, specie nell'anziano, richiede il monitoraggio attento di glicemia e uricemia e va usato con cautela in pazienti con valori di creatininemia $>2 \mathrm{mg} / \mathrm{dL}$.

Infine, la corretta interpretazione della proteinuria superiore al grammo nel paziente iperteso con insufficienza renale cronica può meritare un approfondimento diagnostico anche con biopsia renale. La conoscenza della patologia renale di base infatti può consentire il pieno utilizzo di tutto l'armamentario terapeutico: da un lato, i farmaci antipertensivi come ACE-Inibitori e sartani a scopo anche renoprotettivo e antiproteinurico, dall'altro la terapia specifica della glomerulopatia nelle fasi di danno renale non ancora irreversibile. Per saggiare su scala nazionale il rapporto Rischio/Utilità della biopsia renale anche in questi casi, è in fase di reclutamento attivo lo studio prospettico multicentrico italiano (http://www. statgate.it) agganciato al Registro delle biopsie renali.

\section{Conclusioni}

La prevenzione primaria e secondaria della insufficienza renale cronica da nefroangiosclerosi è rappresentata dallo stretto controllo dei valori pressori e dalla riduzione della proteinuria. In presenza di proteinuria, il controllo pressorio va ottenuto con il blocco del sistema renina-angiotensina anche in pazienti con importante riduzione della funzione renale. La terapia di associazione di $\mathrm{ACE}$ inibitore con sartano non sembra portare vantaggi aggiuntivi ma può essere saggiata in pazienti selezionati con proteinuria persistente superiore al grammo/ die. L'associazione ACE inibitore con antialdosteronico sembra promettere bene ma occorrono ulteriori studi, possibilmente multicentrici.

\section{Riassunto}

La prevenzione primaria e secondaria della insufficienza renale cronica da nefroangiosclerosi è rappresentata dallo stretto controllo dei valori pressori e dalla riduzione della proteinuria. In presenza di proteinuria, il controllo pressorio va ottenuto con il blocco del sistema renina-angiotensina anche in pazienti con importante riduzione della funzione renale. La terapia di associazione di $\mathrm{ACE}$ inibitore con sartano non sembra portare vantaggi aggiuntivi ma può essere saggiata in pazienti selezionati con proteinuria persistente superiore al grammo/ die. L'associazione ACE inibitore con antialdosteronico sembra promettere bene ma occorrono ulteriori studi, possibilmente multicentrici.

Parole Chiave. Prevenzione primaria e secondaria, Ipertensione arteriosa, Rischio cardiovascolare globale, Compliance alla terapia, Terapia personalizzata, Proteinuria

\author{
Indirizzo degli autori: \\ Dr. Simeone Andrulli \\ Divisione di Nefrologia e Dialisi \\ Ospedale 'Alessandro Manzoni' \\ Via dell'Eremo 9 \\ 23900 Lecco \\ s.andrulli@ospedale.lecco.it
}

\section{Bibliografia}

1. Mancia G, De Backer G, Dominiczak A, Cifkova R, et al. 2007 ESH-ESC Practice Guidelines for the Management of Arterial Hypertension: ESH-ESC Task Force on the Management of Arterial Hypertension. J Hypertens 2007; 25(9): 1751-62.

2. Go AS, Chertow GM, Fan D, McCulloch CE, Hsu CY. Chronic kidney disease and the risks of death, cardiovascular events, and hospitalization. New Engl J Med 2004; 351: 1296-305.

3. Sarnak MJ, Greene T, Wang X, Beck G, Kusek JW, Collins AJ, Levey AS. The effect of a lower target blood pressure on the progression of kidney disease: long-term followup of the modification of diet in renal disease study. Ann 
Intern Med 2005; 142(5): 342-51.

4. Imai E, Yamagata $\mathrm{K}$, Iseki $\mathrm{K}$, Iso $\mathrm{H}$, Horio M, Mkino $\mathrm{H}$, Hishida A, Matsuo S. Kidney disease screening program in Japan: history, outcome, and perspectives. Clin J Am Soc Nephrol 2007; 2: 1360-6.

5. Klahr S, Levey AS, Beck GJ, Caggiula AW, Hunsicker L, Kusek JW, Striker G. The effects of dietary protein restriction and blood-pressure control on the progression of chronic renal disease. Modification of Diet in Renal Disease Study Group. N Engl J Med 1994; 330: 877-84.

6. Jafar TH, Schmid CH, Landa M, Giatras I, et al. Angiotensin-converting enzyme inhibitors and progression of nondiabetic renal disease. A meta-analysis of patient-level data. Ann Intern Med 2001; 135: 73-87.

7. Brenner BM, Cooper ME, de Zeeuw D, Keane WF, et al.
Effects of losartan on renal and cardiovascular outcomes in patients with type 2 diabetes and nephropathy. N Engl J Med 2001; 345(12): 861-9.

8. Nakao N, Yoshimura A, Morita H, Takada M, Kayano T, Ideura T. Combination treatment of angiotensin-II receptor blocker and angiotensin-converting-enzyme inhibitor in non-diabetic renal disease (COOPERATE): a randomised controlled trial. Lancet 2003; 361: 117-224.

9. Bianchi S, Bigazzi R, Campese VM. Long-term effects of spironolactone on proteinuria and kidney function in patients with chronic kidney disease. Kidney Int 2006; 70: 2116-23.

10. Hou FF, Zhang X, Zhang GH, Xie D, et al. Efficacy and safety of benazepril for advanced chronic renal insufficiency. N Engl J Med 2006; 354: 131-140. 